\title{
Neurocisticercosis racémica, una rara forma de presentación. Reporte de caso
}

\section{Racemous neurocisticercosis, a rare form of presentation. Case report}

\author{
Diana P. Duarte-Mora ${ }^{1 *}$, Juan E. Muñoz-Montoya ${ }^{1}$, Ma. Camila Vargas-Valdés², Marco L. Charry-López ${ }^{3}$ y \\ Luis O. Rojas-Romero 4 \\ ${ }^{1}$ Departamento de Neurocirugía; ${ }^{2}$ Facultad de Medicina; ${ }^{3}$ Departamento de Neurorradiología; ${ }^{4}$ Departamento de Neurocirugía. Universidad Militar \\ Nueva Granada, Hospital Militar Central, Bogotá, Colombia
}

\section{Resumen}

Antecedentes: La neurocisticercosis es una neuroinfección parasitaria endémica en países en desarrollo causada por Taenia solium. Tiene una presentación clínica variada según la localización y severidad de la infección; puede presentarse de forma intraparenquimatosa, siendo su localización más frecuente la región corticosubcortical, y de forma extra parenquimatosa como quistes racemosos de localización intraventricular o cisternal, que son la presentación menos frecuente. Métodos: El tratamiento de la neurocisticercosis se basa en antiparasitarios como el albendazol para destruir los quistes, controlando la respuesta inflamatoria secundaria con corticosteroide. Se presenta el caso de una paciente de 74 años con cuadro de desorientación, bradipsiquia y alteración en la marcha en quien se identificó por neuroimágenes y estudio de líquido cefalorraquídeo neurocisticercosis racémica asociada a hidrocefalia. Resultados y conclusiones: Recibió manejo médico y quirúrgico, con resolución completa de su sintomatología.

Palabras clave: Neurocisticercosis. Racemosa. Hidrocefalia.

\section{Abstract}

Background: Neurocysticercosis is an endemic parasitic neuroinfection in developing countries caused by Taenia solium. It has a varied clinical presentation according to the location and severity of the infection, and can occur intraparenchymally (with the corticosubcortical region being the most frequent location) and extra-parenchymally (as racemosal cysts with intraventricular or cisternal location, which are the least frequent presentation). Methods: The treatment of neurocysticercosis relies on antiparasitic agents such as albendazole to destroy cysts by controlling the secondary inflammatory response with corticosteroids. We present the case of a 74-year-old patient with disorientation, bradypsychia and gait disturbance in whom it was identified by neuroimaging and study of racemic neurocephalicercosis cerebrospinal fluid associated with hydrocephalus. Results and conclusions: She received medical and surgical management, with complete resolution of her symptomatology.

Key words: Neurocysticercosis. Racemic neurocysticercosis. Neuroinfection.

Correspondencia:

*Diana P. Duarte-Mora

E-mail: dianaduarte05@gmail.com
Disponible en internet: 14-01-2021 Rev Hosp Jua Mex. 2020;87(4):176-180

www.revistahospitaljuarez.com 1405-9622/@ 2020 Sociedad Médico-Quirúrgica del Hospital Juárez de México, A.C. Publicado por Permanyer. Este es un artículo open access bajo la licencia CC BY-NC-ND (http://creativecommons.org/licenses/by-nc-nd/4.0/). 


\section{Introducción}

La neurocisticercosis es un desorden neurológico común causado por una larva con escólex llamada Taenia solium. Puede afectar a cualquier órgano, pero el sitio más común que compromete es el sistema nervioso central (espacio subaracnoideo, ventrículos, médula espinal, parénquima), los ojos y los músculos ${ }^{1,2}$.

La cisticercosis es altamente endémica en países en desarrollo como resultado de su pobre avance económico y la domesticación de $\operatorname{cerdos}^{3}$, aunque en la actualidad se ha observado un incremento en los países desarrollados, debido a la inmigración ${ }^{4}$.

En zonas endémicas como Perú, en donde casi la mitad de la población vive en condiciones en las que la transmisión de $T$. solium es un hecho cotidiano, se ha encontrado una seroprevalencia hasta del $24.2 \%$ con granulomas calcificados como el hallazgo más frecuente en tomografía y se considera que 1 de cada 200 personas tiene convulsiones asociadas a la neurocisticercosis. La carga de la enfermedad no se ha estudiado para varios países de América Latina, sin embargo, extrapolando datos de prevalencia de Perú, se estima que la neurocisticercosis representa alrededor de 400,000 de los casos existentes de enfermedad neurológica sintomática ${ }^{5,6}$.

En la actualidad existen varias formas de presentación de la neurocisticercosis, según García, et al. en dos grupos grandes: forma intraparenquimatosa y extraparenquimatosa. Esta última incluye la forma subaracnoidea (racemosa) e intraventricular ${ }^{7}$. La forma subaracnoidea es la que más se asocia a hidrocefalia, con alta mortalidad sin intervención quirúrgica ${ }^{7}$.

\section{Descripción del caso}

Paciente de sexo femenino de 74 años, sin antecedentes de importancia, con cuadro clínico de un mes de evolución consistente en desorientación, lenguaje incoherente, aumento de la base de sustentación para caminar, bradipsíquica, al examen físico sin ningún síntoma de importancia clínico. Se le realiza resonancia magnética nuclear (RM) simple y contrastada de cerebro, donde se identifican lesiones quísticas en ángulo pontocerebeloso izquierdo y lesión frontal derecha (Fig. 1).

Adicionalmente se realizaron estudios para descartar patología inicialmente infecciosa, identificándose: leucocitos 15,140, neutrófilos $67.10 \%$, hemoglobina $14.8 \mathrm{mg} / \mathrm{dl}$, hematocrito $42.4 \%$, plaquetas 259,000 , glucosa $92 \mathrm{mg} / \mathrm{dl}$, proteína $\mathrm{C}$ reactiva 0.16 y velocidad de sedimentación globular $2 \mathrm{md} / \mathrm{dl}$. Además se realizó punción lumbar, identificándose: presión de apertura $10 \mathrm{cmH}_{2} \mathrm{O}$, líquido transparente, linfocitos $100 \%$, leucocitos 17 células $/ \mathrm{mm}^{3}$, proteínas $45 \mathrm{mg} / \mathrm{dl}$, glucosa $65 \mathrm{mg} / \mathrm{dl}$, adenosina desaminasa 0.9 , prueba serológica para sífilis no reactiva. $Y$ también se llevaron a cabo en líquido cefalorraquídeo (LCR): inmunoglobulina G para cisticerco positiva valor 1.79 por inmunofluorescencia, reacción en cadena de la polimerasa para tuberculosis negativa y detección de antígeno de criptococo en látex negativa.

Siete días posteriores al ingreso, la paciente presenta deterioro neurológico agudo por hidrocefalia obstructiva secundaria a aracnoiditis en cisterna magna (Fig. 2), por lo que se realiza derivación ventriculoperitoneal. Ocho días después, una vez estabilizada la condición clínica de la paciente, se procede a manejo quirúrgico para resección de quistes de ángulo pontocerebeloso y fosa posterior a través de abordaje suboccipital medio, identificándose aracnoiditis en toda la fosa posterior (Fig. 3).

Se envía a patología el tejido resecado y se inicia manejo médico con albendazol $400 \mathrm{mg}$ cada 12 horas por seis semanas y dexametasona $8 \mathrm{mg}$ cada ocho horas por 10 días; la patología de la pared de los quistes resecados reporta presencia de tejido glial asociado a extenso infiltrado inflamatorio agudo. Se realizan imágenes postoperatorias (Fig. 4) en las cuales no se observan quistes en ángulo pontocerebeloso. La paciente evoluciona de forma satisfactoria, con mejoría de su sintomatología neurológica, sin alteraciones al examen físico previo y sin nueva recaída.

\section{Discusión}

La neurocisticercosis es la enfermedad parasitaria más frecuente del sistema nervioso central y la causa más frecuente de epilepsia adquirida. Es causada por el consumo de huevos de Taenia solium, por ruta fecaloral desde el cerdo portador del parásito hacia el humano, que es el portador definitivo ${ }^{8}$. El número exacto de casos de neurocisticercosis en México es desconocido, sin embargo se estima que para el 2015 cerca de medio millón de personas sufrían epilepsia y cefalea severa crónica secundaria a neurocisticercosis, con una carga monetaria asociada con la morbilidad de aproximadamente US\$215,775,056 y con costos por día de hospitalización que oscilan entre US\$2,576 y US\$ $3,487^{9,10}$. Se estima que entre 2009 y 2013 se presentaron 3,626 casos de cisticercosis en Colombia, de los cuales el $57 \%$ correspondieron a neurocisticercosis ${ }^{11}$. 

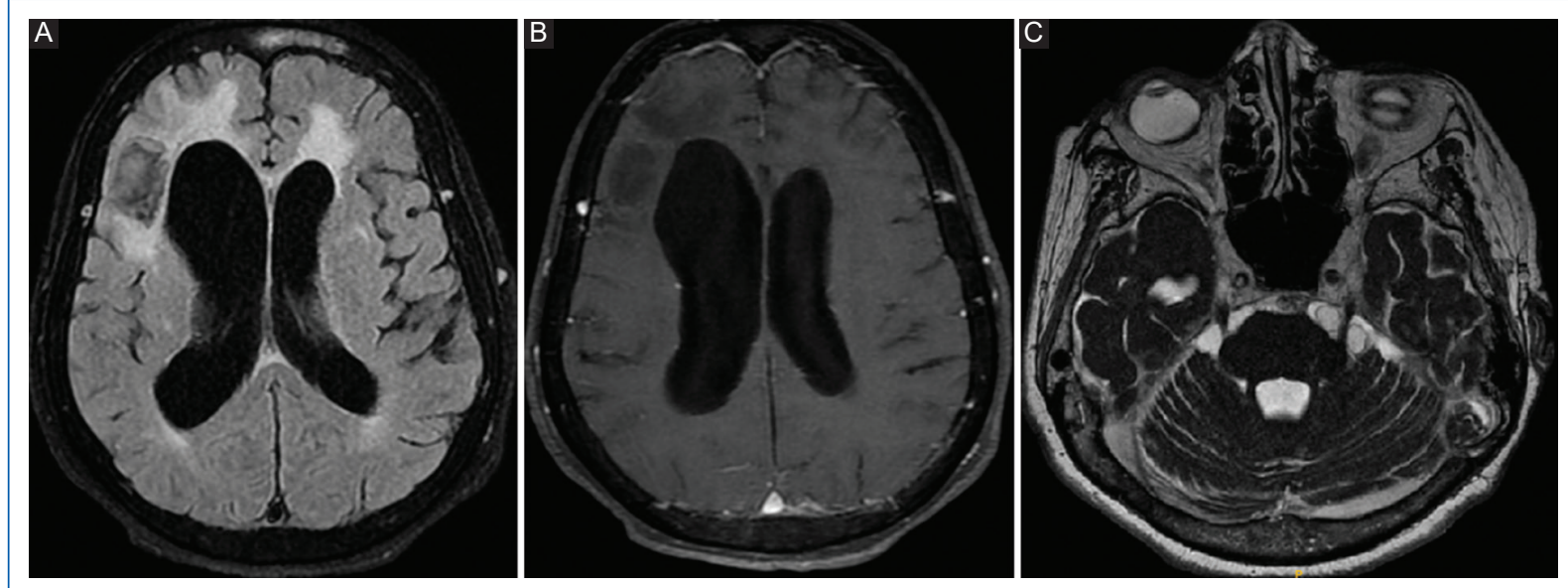

Figura 1. A: resonancia cerebral magnética ponderada en FLAIR con evidencia de lesión frontal derecha hipodensa con halo hiperdenso que genera retracción de cuerno frontal del ventrículo lateral derecho. B: resonancia cerebral magnética ponderada en T1 con medio de contraste que no muestra captación de medio de contraste en lesión frontal derecha. C: resonancia cerebral magnética ponderada en FIESTA con evidencia de lesiones quísticas en ángulo pontocerebeloso adyacentes al quinto nervio craneal izquierdo.

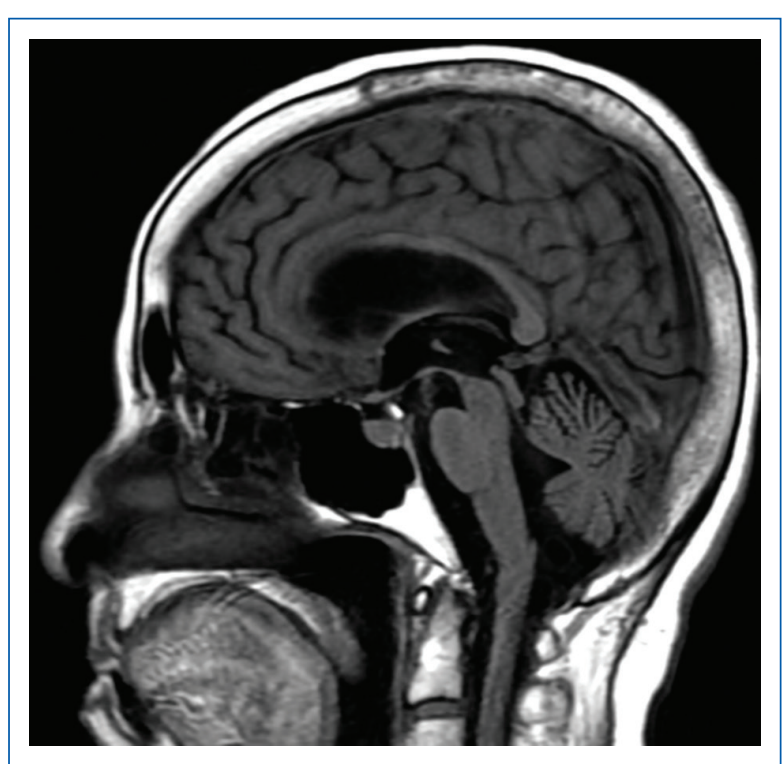

Figura 2. Resonancia cerebral magnética ponderada en FLAIR corte sagital en donde se evidencian lesiones quísticas en cisterna magna, asociado a aumento de tamaño de cavidades ventriculares.

Sus manifestaciones clínicas varían según la localización y severidad de la infección, logrando simular cualquier desorden neurológico, desde el síndrome de BrownSéquard hasta parkinsonismo o psicosis, por lo que se conoce como el gran imitador ${ }^{8}$. Puede presentarse de forma intraparenquimatosa, siendo su localización más frecuente la región corticosubcortical, y de forma extra parenquimatosa como quistes racemosos de localización intraventricular o cisternal, que son la presentación menos frecuente ${ }^{12}$.

El diagnóstico de la neurocisticercosis se basa en las neuroimágenes contrastadas y la evaluación de LCR, incluyendo datos clínicos y epidemiológicos. La prueba de anticuerpos de elección es la electroinmunotransferencia usando glucoproteínas parasitarias que en suero alcanza una sensibilidad del $86 \%$, sin embargo en pacientes con lesiones intraparenquimatosas múltiples, ventriculares o subaracnoideas, como en el caso de nuestra paciente, la sensibilidad es cercana al $100 \%$. La neuroimagen de elección es la RM, donde típicamente las lesiones aparecen hiperintensas en T2 con bordes definidos. La presencia de una sola lesión que muestre el escólex (la cabeza del parásito) es patognomónica para el diagnóstico ${ }^{13,14}$. Se considera que la neurocisticercosis se encuentra activa cuando las lesiones realzan en su periferia a la administración de medio de contraste y es inactiva cuando se evidencian en la imagen lesiones calcificadas. La presencia de eosinofilia en sangre es común, pero no específica; al igual que la presencia de eosinófilos en LCR, puede ser indicativa de neurocisticercosis, aunque este hallazgo ocurre solo en el $15 \%$ de los pacientes ${ }^{15}$.

Los quistes intraventriculares pueden producir hidrocefalia aguda por obstrucción del sistema ventricular en el foramen de Monro, acueducto de Silvio o del cuarto ventrículo, o por degeneración del quiste que produce una respuesta inflamatoria que desencadena 


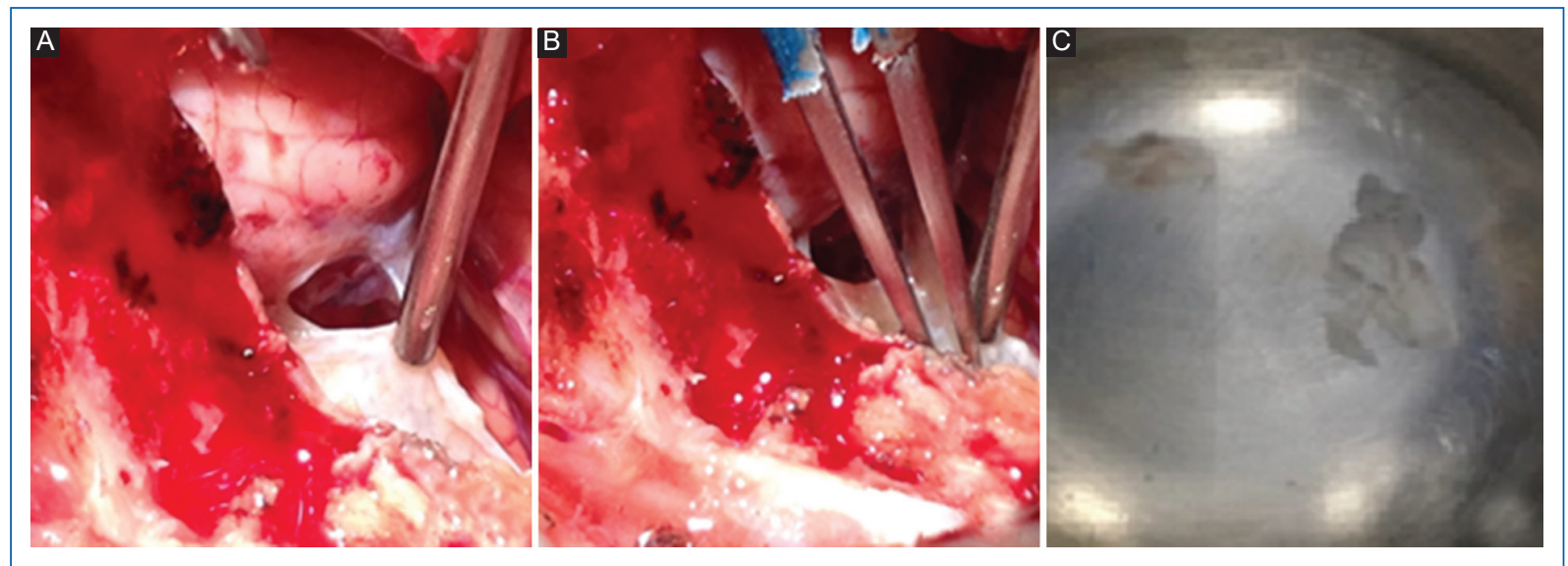

Figura 3. Imágenes intraoperatorias. A: la succión señala áreas de aracnoiditis. B: se realiza resección de aracnoiditis y quistes ubicados en la cisterna magna con ayuda de coagulación bipolar. C: membranas obtenidas y enviadas a estudio histopatológico.

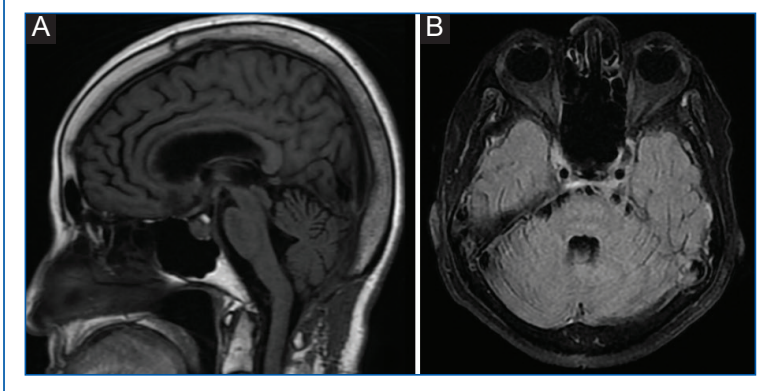

Figura 4. A: resonancia cerebral magnética ponderada en FLAIR corte sagital con disminución de tamaño de cavidades ventriculares y sin evidencia de lesiones quísticas de fosa posterior. B: resonancia cerebral magnética con ausencia de lesiones en ángulo pontocerebeloso izquierdo.

aracnoiditis y vasculitis, presentando así, hidrocefalia de forma subaguda o crónica $^{12}$. La tercera ventriculostomía endoscópica es una opción terapéutica en estos pacientes, que puede mejorar el pronóstico, ya que las derivaciones tienen alta tasa de disfunción, aunque este riesgo puede disminuir con el uso de prednisolona $^{13}$.

El tratamiento antiparasitario no es una intervención urgente y se debe iniciar solamente cuando el paciente se encuentra estable. Los antiparasitarios más comúnmente usados son el albendazol y el praziquantel. El tipo de terapia y la duración están sujetas al número, localización, tamaño y estado de los quistes. El objetivo de la terapia antibiótica es la destrucción de los quistes, adicionando antiinflamatorios como los corticosteroides con el fin de modular la respuesta inmunitaria y disminuir las repercusiones locales y sistémicas de la neurocisticercosis ${ }^{15}$. El tratamiento de la epilepsia en paciente con neurocisticercosis se realiza buscando conseguir el control de crisis con antiepilépticos de primera línea, tales como carbamazepina o fenitoína, los cuales se usan en monoterapia para mejorar la adherencia al tratamiento y teniendo en cuenta las posibles interacciones farmacológicas con el manejo antibiótico que requiere la enfermedad de base; sin embargo, no hay un consenso en cuanto a qué fármaco ni a qué dosis se debe usar para dicho tratamiento ${ }^{16}$.

\section{Conclusión}

En este caso se ilustra una forma de presentación inusual de la neurocisticercosis evidenciando que al instaurar un manejo multidisciplinario y oportuno de la sintomatología y de cada una de las complicaciones de la paciente, se impacta en la respuesta al tratamiento y en el pronóstico neurológico y vital.

\section{Financiamiento}

La presente investigación no ha recibido ayudas específicas provenientes de agencias del sector público, sector comercial o entidades sin ánimo de lucro.

\section{Conflicto de intereses}

Los autores declaran no tener conflicto de intereses alguno. 


\section{Responsabilidades éticas}

Protección de personas y animales. Los autores declaran que para esta investigación no se han realizado experimentos en seres humanos ni en animales.

Confidencialidad de los datos. Los autores declaran que han seguido los protocolos de su centro de trabajo sobre la publicación de datos de pacientes.

Derecho a la privacidad y consentimiento informado. Los autores han obtenido el consentimiento informado de los pacientes y/o sujetos referidos en el artículo.

\section{Bibliografía}

1. Garcia HH, Gonzalez AE, Tsang VC, Gilman RH. Neurocysticercosis: some of the essentials. Pract Neurol. 2006;6(5):288-97.

2. Bastos AL, Marchiori E, Gasparetto EL, Andrade BH, Junior GC, Carvalho RC, et al. Pulmonary and cardiac cysticercosis: helical CT findings. Br J Radiol. 2007;80(951):e58-e60.

3. Garcia $\mathrm{HH}$, del Brutto $\mathrm{OH}$; Cysticercosis Working Group in Peru. Neurocysticercosis: updated concepts about an old disease. Lancet Neurol. 2005;4(10):653-61.

4. Wallin MT, Kurtzke JF. Neurocysticercosis in the United States: review of an important emerging infection. Neurology. 2004;63(9):1559-64.

5. Bern C, Garcia HH, Evans C, Gonzalez AE, Verastegui M, Tsang VC, et al. Magnitude of the disease burden from neurocysticercosis in a developing country. Clin Infect Dis. 1999;29(5):1203-9.
6. Montano SM, Villaran MV, Ylquimiche L, Figueroa JJ, Rodriguez S, Bautista CT, et al. Neurocysticercosis: association between seizures, serology, and brain CT in rural Peru. Neurology. 2005;65(2):229-33.

7. Garcia $\mathrm{HH}$, Nash TE, del Brutto $\mathrm{OH}$. Clinical symptoms, diagnosis, and treatment of neurocisticercosis. Lancet Neurol. 2014;13:1202-15.

8. Wang DD, Huang MC. Cervicomedullary neurocysticercosis causing obstructive hydrocephalus. J Clin Neurosci. 2015;22:1528-31.

9. Bhattarai R, Carabin H, Proaño JV, Flores- Rivera J, Corona T, Flisser A, et al. The monetary burden of cisticercosis in Mexico. PLoS Negl Trop Dis. 2019 Jul 10:13(7):e0007501.

10. Bhattarai R, Carabin H, Flores-Rivera J, Corona T, Proaño JV, Flisser A, et al. Pre-hospitalization, hospitalization, and post-hospitalization costs of patients with neurocysticercosis treated at the Instituto Nacional de Neurologia y Neurocirugia (INNN) in Mexico City, Mexico. Rev Inst Med Trop Sao Paulo. 2018;60:e20.

11. Rodríguez-Morales AJ, Yepes-Echeverri MC, Acevedo-Mendoza WF, Marín-Rincón HA, Culquichicón C, Parra-Valencia E, et al. Mapping the residual incidence of taeniasis and cysticercosis in Colombia, 2009-2013, using geographical information systems: Implications in public health and travel medicine. Travel Med Infect Dis. 2018;22:51-7.

12. Mahale RR, Mehta A, Rangasetty S. Extraparenchymal (racemose) neurocysticercosis and its multitude manifesttions: A comprehensve review. J Clin Neurol. 2015;11(3):203-11.

13. Fogang YF, Savadogo AA, Camara M, Toffa DH, Basse A, Sow AD, et al. Managing neurocysticercosis: challenges and solutions. Int J Gen Med. 2015;8:333-44.

14. White AC Jr, Coyle CM, Rajshekhar V, Singh G, Hauser WA, Mohanty A, et al. Diagnosis and Treatment of Neurocysticercosis: 2017 Clinical Practice Guidelines by the Infectious Diseases Society of America (IDSA) and the American Society of Tropical Medicine and Hygiene (ASTMH). Clin Infect Dis. 2018;66(8):e49-e75.

15. Krupa K, Krupa K, Pisculli ML, Athas DM, Farrell CJ. Racemose neurocysticercosis. Surg Neurol Int. 2016;7:12.

16. Bustos JA, García HH, Del Brutto $\mathrm{OH}$. Antiepileptic drug therapy and recommendations for withdrawal in patients with seizures and epilepsy due to neurocysticercosis. Expert Rev Neurother. 2016;16(9):1079-85. 